\title{
Adaptación al español y validación de criterio de una escala para la tamización de problemas emocionales y del comportamiento en la primera infancia
}

\author{
Luz Helena Cano ${ }^{1,2}$, María Natalia Acosta ${ }^{2}$, Adriana Pulido1,2 \\ 1 Red Social de Conocimiento por un Inicio Parejo de la Vida, Bogotá, D.C., Colombia \\ 2 Eje de Salud Pública, Fundación Santa Fe de Bogotá, Bogotá, D.C., Colombia \\ Introducción. La detección temprana del riesgo de problemas emocionales y del comportamiento en \\ niños puede contribuir al desarrollo de estrategias que promuevan la salud mental desde la primera \\ infancia. En Colombia no existe una herramienta validada para dicha detección. \\ Objetivos. Seleccionar, adaptar y establecer la validez de criterio de una escala de tamización de \\ problemas emocionales y del comportamiento en niños menores de seis años. \\ Materiales y métodos. A partir de una revisión de la literatura y un consenso de expertos, se seleccionó \\ la herramienta Early Childhood Screening Assessment (ECSA). Posteriormente, se llevó a cabo su \\ adaptación lingüística y se determinó la validez de criterio mediante una curva de características de \\ recibidor-operador (Receiver Operating Characteristic, ROC), y se la comparó con el cuestionario Child \\ Behavior Checklist (CBCL 1,5-5). En el estudio participaron 206 cuidadores de niños entre el año y \\ medio y los seis años de edad de la ciudad de Tunja y el municipio de Sopó. \\ Resultados. La puntuación del ECSA presentó una buena correlación con la puntuación $t$ total del \\ CBCL 1,5-5 (ro de Spearman=0,75; $p<0,01$ ). La escala ECSA tuvo una sensibilidad de $86 \%$ y una \\ especificidad de $82 \%$ al establecer un punto de corte de 24 para la población estudiada. \\ Conclusión. En este primer estudio de adaptación y validación de la versión en español de la escala \\ ECSA, se detectaron buenos valores de sensibilidad y especificidad para la tamización de problemas \\ emocionales y del comportamiento en la primera infancia.
}

Palabras clave: conducta; emociones; preescolar; escala de evaluación de la conducta; tamización masiva; estudios de validación.

doi: https://doi.org/10.7705/biomedica.v38i0.3795

\section{Adaptation to Spanish and criterion validity of a scale for the screening of emotional and behavioral problems in early childhood}

Introduction: Early identification of emotional and behavioral risk in children can contribute to the development of strategies that promote mental health from early childhood. In Colombia, we do not count with a validated screening tool for emotional and behavioral problems in this population.

Objectives: To identify, adapt, and establish evidence for the criterion validity of a screening tool for emotional and behavioral problems in less than 6-year-old children.

Materials and methods: We selected the Early Childhood Screening Assessment (ECSA) after a literature review and expert consensus and then we undertook its linguistic adaptation. For criterion validity, we used the Receiver Operating Characteristic (ROC) comparing the scale with the Child Behavior Checklist (CBCL 1,5-5). The sample included 206 caregivers of children between 1.5 and 6 years of age from the city of Tunja and the municipality of Sopó.

Results: The ECSA showed a good correlation with the total t-score of the CBCL 1,5-5 (Spearman rho= $0,75, p<0,01)$. The scale showed a sensitivity of $86 \%$ and a specificity of $82 \%$ at a cut-off point of 24 .

Conclusion: In this first adaptation to Spanish and validation study of the ECSA scale, we found good sensitivity and specificity values for the screening of emotional and behavioral problems in early childhood.

Key words: behavior; emotions; child preschool; behavior rating scale; mass screening; validation studies. doi: https://doi.org/10.7705/biomedica.v38i0.3795

\footnotetext{
Contribución de los autores:

Luz Helena Cano: diseño del protocolo del estudio; revisión de la literatura; diseño, consecución y análisis de resultados del consenso de expertos; diseño, coordinación y participación en la recolección, digitación y análisis de datos para el proceso de validación; aporte de referencias del protocolo y búsqueda bibliográfica

María Natalia Acosta: participación en la recolección, digitación y análisis de datos para el proceso de validación

Adriana Pulido: diseño conceptual del protocolo de estudio

Todas las autoras participaron en la escritura del manuscrito.
} 
La detección temprana del riesgo de problemas emocionales o del comportamiento en niños es una de las prioridades actuales de los servicios de salud mental (1). En la atención pediátrica primaria, el desarrollo y la adquisición de competencias sociales y emocionales de los niños constituyen un hito fundamental. En el contexto de las funciones ejecutivas, se ha demostrado la estrecha relación entre la regulación emocional y la inhibición del comportamiento, y los resultados cognitivos y psicosociales inmediatos y futuros en los niños (2-5).

La salud mental en la infancia temprana (desde el nacimiento hasta los seis años), se entiende como la capacidad que tiene el niño de experimentar, regular y expresar sus emociones a partir de las relaciones interpersonales cercanas y seguras, y de explorar su ambiente y aprender. La salud mental en la niñez temprana se relaciona con un buen desarrollo emocional y social (6).

Entre los 18 meses y los cinco años de vida, se establecen los mecanismos fundamentales de control emocional y del comportamiento, incluida la capacidad para regular la ira, manejar la frustración e inhibir reacciones verbales o físicas frente a estímulos negativos. Sin embargo, en algunos niños persisten las conductas desafiantes, la agresividad y la irritabilidad, lo cual incide negativamente en su desarrollo y aprendizaje. Alrededor del $5 \%$ de los niños con conductas de oposición a los tres años de edad persisten en ellas durante la edad escolar (7).

Los padres detectan que las principales causas de deserción en la primaria son los frecuentes conflictos entre pares, las dificultades en las relaciones entre profesores y alumnos, y los problemas de agresión e intimidación escolar (8). Según la encuesta de deserción escolar del Departamento Nacional de Planeación (DNP) y el Programa de Naciones Unidas para el Desarrollo (PNUD) realizada en algunos departamentos de Colombia (2002-2003), en el $61 \%$ de los casos el abandono de la escuela se debe a problemas de conducta o falta de motivación y en el $13 \%$, al bajo rendimiento académico, lo cual coincide con estudios en otros países, en los que se ha encontrado que los problemas de conducta están asociados con la deserción escolar (8).

\footnotetext{
Correspondencia:

Luz Helena Cano, Carrera 7B N 123-90, piso 3, Bogotá, D.C., Colombia

Teléfono: (571) 603 0303, extensión 5715

canoluzhelena@gmail.com
}

Recibido: 16/02/17; aceptado: 12/09/17
Los estudios más representativos sobre psicopatología en lactantes y preescolares se han hecho en países de ingresos altos, y las tasas de prevalencia y comorbilidad se han estimado para poblaciones predominantemente norteamericanas, europeas y australianas $(9,10)$. En ellos, se ha estimado una prevalencia de trastornos en preescolares entre 7 y $25 \%(7,11)$. Sin embargo, en un estudio multicéntrico en veintitrés países de cuatro continentes (incluida Suramérica), se encontró que, a pesar de la enorme variación en la crianza de los niños y en las diferencias sociales de los países, los patrones de síntomas reportados por los padres en todas las sociedades eran similares. A partir de esta concordancia, se planteó un modelo de siete dimensiones básicas o síndromes psicopatológicos que serviría para establecer una taxonomía válida y confiable de los problemas emocionales y de comportamiento en la primera infancia $(12,13)$.

Los trastornos descritos en la edad preescolar incluyen el déficit de atención e hiperactividad, el retraso global del desarrollo, los trastornos del espectro autista, la ansiedad por separación y el mutismo selectivo en la categoría de trastornos de ansiedad, el trastorno de vinculación reactiva en la categoría de trastornos por trauma y estrés, los trastornos específicos del aprendizaje, los motores, el trastorno de oposición desafiante, el trastorno de estrés postraumático (subtipo preescolar) y los trastornos del estado de ánimo, especialmente el depresivo (7,14-16). Otra forma de describir los síntomas de estos trastornos es la clasificación en síndromes de 'internalización', que abarcan el espectro emocional, y de 'externalización', es decir, los síntomas relacionados con la conducta.

En Latinoamérica, se cuenta con estudios en niños a partir de los tres años hasta la adolescencia (1722). En la Encuesta Nacional de Salud Mental del 2015 en Colombia, se indagó sobre los trastornos únicamente en niños de 7 a 11 años (23), y en la revisión hecha no se encontraron estudios sobre tamización de problemas emocionales y de comportamiento en población lactante y preescolar. La mayoría de los estudios en población latinoamericana se centran en intervenciones en niños internados en instituciones y relacionados con las formas de crianza y apego (24-26). Existe una brecha evidente entre la cantidad de estudios sobre la salud mental en la primera infancia en países como Estados Unidos, Australia y los europeos, y en el resto del mundo (9). 
En Colombia, la investigación sobre problemas emocionales y de conducta en niños menores de seis años es escasa, pues la mayoría de estudios sobre psicopatología infantil se han centrado en el déficit de atención e hiperactividad en población escolar (27-29). En otros estudios, se ha analizado la vulnerabilidad de la primera infancia en términos de salud mental y violencia en el país y se describe la asociación entre el trastorno de estrés postraumático en la primera infancia y el desplazamiento. Según los resultados, la exposición a la guerra aumentó el riesgo de problemas emocionales y del comportamiento, así como el de deterioro de su salud mental (30).

En los estudios longitudinales se ha evidenciado que los estados sintomáticos tempranos están asociados con el desarrollo posterior de trastornos psiquiátricos $(7,31)$, y se ha estimado un riesgo dos a seis veces mayor de presentar en la edad adulta condiciones descritas en el "Manual diagnóstico y estadístico de los trastornos mentales" (Diagnostic and Statistical Manual of Mental Disorders, DSM). Se ha demostrado que con la tamización en programas de atención primaria y secundaria se logra detectar una cuarta parte de los niños que, posteriormente, tienen un diagnóstico psicopatológico (32-35). Hasta donde se sabe, en el país no se cuenta con una herramienta validada que permita hacer la tamización de problemas emocionales y del comportamiento en la primera infancia.

Con la intención de contribuir a la detección temprana de los riesgos frente a dichos problemas, así como a su estudio en el país, el objetivo del presente estudio fue seleccionar, adaptar y determinar la validez de criterio de una escala seleccionada mediante una revisión de la literatura y un consenso de expertos para la tamización de tales problemas en niños menores de seis años en dos municipios de los departamentos de Boyacá y Cundinamarca.

\section{Materiales y métodos}

\section{Diseño}

Se hizo un estudio instrumental de corte transversal en tres fases (36). En la primera, se revisó la literatura científica y se hizo un consenso de expertos para la selección del instrumento; en la segunda, este se tradujo y se hizo su adaptación lingüística, y en la tercera, se hizo la validación de criterio.

El estudio fue aprobado por el Comité de Ética de la Universidad de la Sabana (2012). La primera y la segunda fases se desarrollaron como proyecto de investigación durante el año de Servicio Social
Obligatorio en Medicina (modalidad investigación, Resolución 540 del 27 de junio de 2012) en el Centro de Estudios e Investigación en Salud (CEIS) del Hospital Universitario Fundación Santa Fe de Bogotá entre junio de 2012 y agosto de 2013, en el marco del programa de investigación de la Unión Temporal Inicio Parejo de la Vida (IPV,http:// www.inicioparejodelavida.org/) y como soporte instrumental para la caracterización del estado de salud y el desarrollo de los niños del programa. La tercera fase fue financiada por Colciencias (20142015) mediante la convocatoria 17 del programa de Jóvenes investigadores e innovadores 2013.

\section{Primera fase: revisión de la literatura}

Entre mayo y julio de 2012, se hizo la revisión de la literatura para seleccionar herramientas de tamización de problemas emocionales y del comportamiento en niños menores de seis años. La búsqueda se hizo en Medline (PubMed) y Google Scholar, y se consideraron trabajos en inglés y español. Se encontraron catorce instrumentos, de los cuales se escogieron los que cumplían con los criterios de selección para el consenso de expertos.

Se consideraron los siguientes criterios de inclusión: que su propósito fuera la tamización; que fueran de rango amplio en los dominios emocional y de comportamiento; que se hubieran diseñado para niños de 0 a 5 años (o subgrupos etarios); que fueran de libre acceso o reproducción sin costo, y que su calificación e interpretación estuvieran a cargo de diferentes profesionales de la salud.

Los criterios de exclusión fueron los siguientes: que su rango fuera estrecho, por ejemplo, que contemplaran un solo dominio o un trastorno específico (exclusivas para déficit de atención e hiperactividad, trastornos de ansiedad, autismo, etc.); que contemplaran múltiples dominios (emocional, del comportamiento, motor o de lenguaje); que fueran entrevistas o escalas diagnósticas, y aquellos sobre los que no se obtuvo respuesta del autor o la editorial (un mes de plazo y dos intentos de contacto).

Aplicados estos criterios, se seleccionaron los siguientes instrumentos: Pediatric Symptom Checklist (PSC) (37), Ages and Stages Questionnaire Social-Emotional (ASQ-SE) (38), Early Childhood Screening Assessment (ECSA) (39) y Strength and Difficulties Questionnaire (SDQ 3-4) (33).

\section{Consenso de expertos}

Este se llevó a cabo entre febrero y junio de 2013, utilizando el método Delphi en línea (40-43). La 
convocatoria se hizo mediante cartas de invitación a los especialistas. Después de dos intentos de contacto y llamadas telefónicas, nueve expertos consintieron en participar (tasa de respuesta: $33 \%$ ): cinco psiquiatras de niños y adolescentes, tres psicólogos con experiencia en psicometría, salud pública o niñez y un psiquiatra general, vinculados a diferentes instituciones de salud o de educación de Bogotá y Medellín. La participación fue voluntaria y no se ofreció remuneración económica. Todos los expertos participaron durante las tres rondas del consenso.

Tras enviar los instrumentos con su correspondiente traducción (cuando se disponía de ella) y un resumen de las características y propiedades psicométricas en las dos rondas iniciales, se solicitó a los expertos calificar los siguientes aspectos en cada instrumento mediante un cuestionario estructurado: cumplimiento de la función de tamización; evaluación de constructos emocionales y de comportamiento (validación de constructo y de contenido); idoneidad para el rango de edad y la población de cuidadores; tiempo de respuesta (menos de 20 minutos); puntuación e interpretación sencillas; viabilidad para la validación cultural; consecuencia con los objetivos de la investigación, y calificación global del instrumento.

Se utilizaron escalas de Likert de cinco puntos ("muy en desacuerdo" a "muy de acuerdo"; "muy inapropiado" a "muy apropiado"; "muy deficiente" a "excelente") y un espacio final para comentarios. En una tercera ronda se hizo la realimentación con los resultados de las rondas iniciales y se solicitó la selección de uno de los instrumentos.

En el análisis de los datos, se calcularon las medidas de tendencia central para las dos rondas iniciales: SDQ 3-4 $(=3,85 ; M=4,0)$; ECSA $(=4,17$; $M=4,0)$; ASQ-SE $(=3,86 ; M=4,0)$, y PSC $(=3,82$; $M=4,0)$. En la tercera ronda, se calcularon las frecuencias y los porcentajes de preferencia para cada instrumento: $37,5 \%$, ECSA; $37,5 \%$, ASQSE; $12,5 \%$, SDQ 3-4, y $12,5 \%$, PSC.

Analizadas las tres rondas, se compararon en detalle el ECSA y el ASQ-SE, y se estudiaron los resultados cuantitativos y las valoraciones cualitativas de las dos primeras rondas. EI ECSA obtuvo una mejor calificación de los expertos en cuanto a la función de tamización ( $E C S A \bar{x}=4,55 ; M=5,0$ Vs. ASQ-SE $\bar{x}=4,11 ; M=4,0)$, diligenciamiento por parte de los cuidadores (ECSA $\bar{x}=4,44 ; M=4,0 \mathrm{Vs}$. ASQ-SE $\bar{x}=3,66 ; M=3,0$ ), brevedad del tiempo para responderlo ( $E C S A \bar{x}=4,0 ; M=4,0$ Vs. $A S Q$ -
SE $\bar{x}=3,33 ; M=4,0$ ), viabilidad de la validación cultural (ECSA $\bar{x}=4,0 ; M=4,0$ Vs. ASQ-SE $\bar{x}=3,55$; $M=3,0)$ y concordancia con los objetivos (ECSA $\bar{x}$ $=4,44 ; M=5,0 \mathrm{Vs}$. ASQ-SE $\bar{x}=3,77 ; M=4,0)$. El único criterio en el que el ASQ-SE puntuó mejor que el ECSA fue en el rango de edad de los evaluados ( $E C S A \bar{x}=4,33 ; M=4,0$ Vs. ASQ-SE $\bar{x}=4,55 ; M=5,0)$.

Las apreciaciones cualitativas incluyeron las siguientes: "el ECSA debe ser correctamente traducido y ajustado a la terminología local"; "el ECSA resulta sencillo y apropiado para una tarea de tamizaje (sic)"; "de manera particular prefiero ASQ-SE por los rangos de edad que maneja, el diseño y las propiedades psicométricas, pero para los demás aspectos el ECSA es una mejor opción"; "el ECSA es un instrumento sencillo de entender, con preguntas cortas y que se puede adquirir en la red sin costo adicional para las instituciones y no necesita ningún entrenamiento especial para calificarla".

Con base en estos resultados, se seleccionó el Early Childhood Screening Assessment (ECSA) como el instrumento para adaptar y validar en esta investigación.

\section{Segunda fase: traducción y adaptación lingüística}

El proceso incluyó la traducción, la traducción inversa y la revisión (44-48). La traducción al español estuvo a cargo de un traductor certificado (TR1) y un profesional bilingüe familiarizado con el tema de interés (TR2). Posteriormente, se discutieron las diferencias (uso de sinónimos, términos poco usados en el lenguaje cotidiano, diferentes estructuras en las frases o lenguaje complejo) y se construyó una versión final (TR1 + TR2) (45).

La traducción inversa la hicieron de forma independiente dos personas bilingües y sin conocimiento teórico. Las dos traducciones se compararon con el instrumento original y no se encontraron grandes diferencias semánticas. Mediante este ejercicio, se completó el proceso de equivalencia técnica o traducción literal (48).

En cuanto a la revisión, se evaluó la equivalencia conceptual de la traducción con el apoyo de un médico psiquiatra con formación en psiquiatría infantil. Se determinó si los ítems de la versión traducida se referían a los mismos constructos y dominios que la versión original. Cuando no hubo claridad sobre lo que el dominio pretendía medir, se consultó con los creadores de la escala. Las modificaciones sugeridas en los ítems en el proceso de revisión, se tuvieron en cuenta para la construcción de una nueva versión del instrumento original. 
En lo concerniente a la comprensión y relevancia cultural, siete profesionales de la salud (medicina, nutrición, terapia ocupacional, epidemiología y psicología) y una secretaria revisaron el instrumento para evaluar la claridad del enunciado, la pertinencia lingüística para la población objetivo y la conveniencia para la lectura de personas con un nivel educativo de quinto de primaria como máximo (46). En un grupo focal se discutieron y acordaron los ajustes lingüísticos necesarios y se construyó el instrumento para la prueba piloto.

\section{Tercera fase: validez de criterio}

El proceso de validación de criterio incluyó la aplicación del instrumento sujeto a validación y su comparación frente a la prueba de referencia (49).

Prueba piloto: en una reunión semanal de beneficiarios de programas sociales de la alcaldía municipal de Tunja, se invitó a las madres, padres o cuidadores principales de niños menores de seis años a participar en el estudio.

El grupo inicial incluyó 22 personas, de las cuales 16 aceptaron participar. De estas, cinco no cumplían con los criterios de selección (cuadro 1), por lo que la muestra final incluyó once cuidadores. Se organizaron dos grupos focales para el desarrollo del ejercicio. Una vez firmado el consentimiento informado, se entregó un formato sociodemográfico $y$, después, los instrumentos ECSA y CBCL 1,5-5 (prueba de referencia definida para la tercera fase). Cuando los participantes terminaron de responder, se discutió con cada cuidador si había tenido dificultad para comprender alguno de los enunciados o si se había sentido incómodo al responder. Se registraron los tiempos de respuesta a las preguntas y las observaciones de los participantes.

Participantes: se utilizaron 218 conjuntos de instrumentos (sociodemográfico, ECSA y CBCL 1,5-

$5)$ en padres, madres $O$ abuelas de niños entre

Cuadro 1. Criterios de inclusión y exclusión de los participantes en el estudio de validación

\begin{tabular}{l}
\hline Criterios de inclusión \\
\hline El niño estaba entre el año y medio y los 5 años y 11 meses \\
de edad \\
Madre, padre o cuidador mayor de 18 años \\
El niño asistía al centro de cuidado o centro educativo \\
Residente en los departamentos de Cundinamarca o Boyacá \\
\hline Criterios de exclusión
\end{tabular}

Niño con discapacidad sensorial (sordera, ceguera) Niño diagnosticado con autismo, trastorno del desarrollo o del lenguaje, parálisis cerebral o epilepsia uno y medio y cinco años inscritos en instituciones educativas de Tunja y Sopó durante el 2014 y el 2015. Los criterios de selección se presentan en el cuadro 1.

\section{Muestreo}

Se seleccionaron por conveniencia jardines infantiles y centros de desarrollo integral adscritos al Instituto Colombiano de Bienestar Familiar (ICBF) de la ciudad de Tunja $(n=4)$ y el municipio de Sopó $(n=3)$.

\section{Tamaño de la muestra}

La muestra total incluyó 218 cuidadores; se emplearon los criterios de validación psicométrica de instrumentos reportados en la literatura (al menos, cinco encuestados por cada ítem del cuestionario) $(50,51)$. Además, el tamaño de la muestra se confirmó mediante la estimación de proporciones, con una proporción de niños con remisión de $50 \%$, y un mínimo de 196 padres y cuidadores, para un error de muestreo de $7 \%$. Se excluyeron 12 participantes que no completaron las encuestas.

\section{Procedimientos}

Tanto en Tunja como en Sopó, se invitó a los padres y cuidadores que cumplían con los criterios de selección a participar en el estudio y se los citó a una reunión para que respondieran los cuestionarios. Se solicitó su consentimiento informado antes de iniciar el estudio. Las escalas ECSA y CBCL 1,5-5 se entregaron para ser diligenciadas durante la misma reunión. Todos los cuestionarios estaban impresos y se revisaron manualmente para verificar que estuvieran completos.

\section{Instrumentos}

Se utilizó un cuestionario sociodemográfico para recopilar la información básica sobre el niño, el estrato socioeconómico, la afiliación a la seguridad social, las enfermedades y los antecedentes familiares de enfermedad mental, así como la información básica sobre el cuidador que respondió las escalas (edad, escolaridad, relación con el niño).

Early Childhood Screening Assessment (ECSA). Esta escala permite la tamización de los problemas emocionales o de comportamiento de niños entre el año y medio y los cinco años de edad. Está compuesta por 40 preguntas y una escala Likert de tres puntos, y la respuesta a las preguntas toma de 5 a 7 minutos. Es visualmente sencilla, rápida de calificar (menos de un minuto) y se puede utilizar 
como un primer paso para detectar a los niños con mayor riesgo de problemas de salud mental (39). Se diseñó específicamente para abordar los desafíos logísticos del ámbito pediátrico (40).

El estudio original concluyó que la ECSA era una escala fácil de usar, con validez convergente, validez de criterio y una excelente fiabilidad pruebareprueba (sensibilidad=86\%; especificidad $=83 \%$; consistencia interna $=0,91$; prueba de fiabilidad a los 10 días con ro de Spearman $=0,81 ; p \leq 0,01)(47)$. Su propósito es detectar los síntomas de base de trastornos de internalización, externalización, reguladores, del desarrollo y 'relacionales' (39).

La escala se deriva del DSM-IV, de los criterios diagnósticos de investigación (Research Diagnostic Criteria, RDC) y de la clasificación de la organización ZERO TO THREE (39). Está compuesta por 36 ítems relacionados con el desarrollo emocional y del comportamiento, y por cuatro preguntas que indagan sobre la depresión materna. El uso y la reproducción de la herramienta son gratuitos (39).

Child Behavior Checklist 1,5-5.La CBCL 1,5-5 es una herramienta reconocida que se utiliza en estudios epidemiológicos y clínicos. Es el instrumento de referencia en múltiples estudios de validación y uno de los instrumentos utilizados en el desarrollo original del cuestionario ECSA $(39,53)$. Cuenta con una versión validada en español para población latina, con grupos normativos para Perú y Chile, y la posibilidad de una puntuación multicultural $(39,53,55)$.

Es un cuestionario de 99 preguntas que evalúa problemas sociales y emocionales en tres dominios (internalización, externalización y puntuación total) (55). También, ofrece puntuaciones a partir de escalas derivadas del DSM. La calificación establece puntos de corte para la tamización subclínica (puntuación $\mathrm{t} \geq 60$ ) y clínica (puntuación $\mathrm{t} \geq 63$ ) (11). La organización ASEBA (www.aseba.org) aprobó la licencia para su reproducción en este proyecto.

\section{Análisis estadístico}

Se construyó una matriz para diligenciar la información sociodemográfica y la escala ECSA. EI CBCL se diligenció en el software de la organización. Ambos instrumentos se digitaron por duplicado y se procesaron en Stata ${ }^{\mathrm{TM}} 12,4$ o SPSS ${ }^{\mathrm{TM}}$ 23.0.

Las variables cualitativas se resumieron en frecuencias y porcentajes. En las variables cualitativas se validó la normalidad (prueba de ShapiroWilk); si presentaban un comportamiento normal, se expresaban como promedios y desviaciones estándar, y si no, se calcularon como medianas y rangos intercuartílicos (RI) (56).

Para determinar la asociación con las variables cualitativas, se utilizaron la prueba de ji al cuadrado o el test de Fisher, según el caso, y para las asociaciones de variables cuantitativas, se utilizó la prueba t de Student o la U de Mann-Whitney (57).

Para establecer la validez de criterio, se estimaron la sensibilidad, la especificidad, el valor predictivo positivo y el negativo, y las razones de máxima verosimilitud positiva y negativa del cuestionario ECSA comparado con el instrumento de referencia CBCL 1,5-5 (52,58). En el CBCL 1,5-5, se tomó como tamización positiva una puntuación t mayor o igual a 60 (11).

En las dos escalas, se utilizó la puntuación total del instrumento para cualquier tipo de trastorno. Se calculó el área bajo la curva (ROC), y se fijó el punto de corte donde se optimizaban la sensibilidad y la especificidad (59). Se calculó el coeficiente de Spearman para estimar la correlación entre las dos escalas (60).

\section{Resultados}

\section{Población}

Los niños participantes tenían una mediana de edad de 47 meses (RI=33 a 53); el 8,3\% $(n=17)$ tenía entre 17 y 23 meses, el $18,4 \%(n=38)$, entre 24 y 35 meses, el 25,2\% ( $n=52)$, entre 36 y 47 meses, y el 48,1\% ( $n=99)$, entre 48 y 65 meses; el $54,9 \%(n=113)$ era de sexo masculino, y el $99,5 \%$ estaba afiliado a algún régimen de salud; el $83 \%$ $(n=171)$ pertenecía a los estratos socioeconómicos 1 y 2 , y el $14,6 \%(n=30)$, a los estratos 3 y 4 . Los cuidadores reportaron antecedentes familiares de enfermedad mental en $7,3 \%(n=15)$ de los niños participantes.

La mediana de edad de los cuidadores participantes fue de 29 años (RI: 24 a 34); $93 \%(n=191)$ de ellos eran las madres, $5 \%(n=11)$, los padres, y $2 \%$ $(n=4)$, las abuelas. El $16 \%(n=33)$ de los cuidadores había cursado la primaria (completa o incompleta), el $52 \%$ ( $n=107)$, la secundaria (completa o incompleta), y el $32 \%(n=66)$, estudios superiores (técnica, pregrado o posgrado) (cuadro 2).

\section{Puntuación en la escala ECSA}

La puntuación mediana fue de 20 puntos $(R \mid=15$ a 28), en un rango de 1 a 53 sobre 74 puntos posibles. En cuanto a la calificación dada a cada uno 
Cuadro 2. Resultados sociodemográficos

\begin{tabular}{|c|c|c|}
\hline Niños & $\%$ & $\mathbf{n}$ \\
\hline \multicolumn{3}{|l|}{ Edad (meses) } \\
\hline Entre 17 y 23 & 8,3 & 17 \\
\hline Entre 24 y 35 & 18,4 & 38 \\
\hline Entre 36 y 44 & 25,2 & 52 \\
\hline Entre 48 y 65 & 48,1 & 99 \\
\hline Mediana & \multicolumn{2}{|c|}{$47(\mathrm{R})=33$ a 53} \\
\hline \multicolumn{3}{|l|}{ Sexo } \\
\hline Masculino & 54,9 & 113 \\
\hline Femenino & 45,1 & 93 \\
\hline Total & & 206 \\
\hline \multicolumn{3}{|l|}{ Estrato socioeconómico } \\
\hline Estrato 1-2 & 83 & 171 \\
\hline Estrato 3-4 & 14,6 & 30 \\
\hline No sabe/no responde & 2,4 & 5 \\
\hline \multicolumn{3}{|l|}{ Afiliados al régimen de salud } \\
\hline Sí & 99,5 & \\
\hline No & 5 & \\
\hline Cuidadores & $\%$ & $\mathbf{n}$ \\
\hline Edad mediana (años) & \multicolumn{2}{|c|}{$29(\mathrm{RI}=24$ a 34$)$} \\
\hline \multicolumn{3}{|l|}{ Rol } \\
\hline Madre & 93 & 191 \\
\hline Padre & 5 & 11 \\
\hline Abuela & 2 & 4 \\
\hline \multicolumn{3}{|l|}{ Nivel educativo } \\
\hline Primaria (completa o incompleta) & 16 & 33 \\
\hline Secundaria (completa o incompleta) & 52 & 107 \\
\hline $\begin{array}{l}\text { Estudios superiores (técnica, pregrado } \\
\text { o posgrado) }\end{array}$ & 32 & 66 \\
\hline \multicolumn{3}{|l|}{$\begin{array}{l}\text { Reportó antecedentes familiares de } \\
\text { enfermedad mental }\end{array}$} \\
\hline Sí & 7,3 & 15 \\
\hline No & 87,4 & 180 \\
\hline No sabe o no responde & 5,3 & 11 \\
\hline
\end{tabular}

de los ítems del instrumento, las medianas más altas las obtuvieron los que medían la inquietud (es muy inquieto, siempre está haciendo algo o buscando algo para hacer), en tanto que las medianas más bajas fueron las correspondientes a los comportamientos repetitivos (balanceo, aleteo) y al interés sexual (se interesa en exceso por juegos sexuales o partes del cuerpo).

En cuanto a la depresión materna, todas las madres respondieron a las preguntas sobre esta condición, y $45 \%(n=87)$ de ellas reportaron por lo menos un ítem para depresión materna (1 de 8 puntos posibles).

\section{Validación de criterio}

El coeficiente de correlación mostró una correlación positiva entre la escala ECSA y el cuestionario CBCL 1.5-5 (ro de Spearman=0,75; $p<0,01$ ). El área bajo la curva fue de 0,91 (figura 1), con una razón de máxima verosimilitud positiva de 4,8
$\left(\mathrm{IC}_{95 \%}: 4,4-5,2\right)$ y una negativa de $0,2\left(\mathrm{IC}_{95 \%}: 0,13-\right.$ 0,23 ). En el cuadro 3 se muestran los índices de validez de criterio en la población estudiada con el punto de corte sugerido y, en el cuadro 4, con otros puntos de corte. El resultado en la ECSA fue positivo en $34 \%(n=70)$ de los niños. En 20,4\% $(n=42)$ de los niños se confirmó con el CBCL 1,5-5 la valoración positiva del riesgo registrada mediante el cuestionario ECSA; el 3,4 \% (n=7) de los niños con resultados positivos en el CBCL1,55, no fue detectado por el cuestionario ECSA.

Se encontró una relación estadísticamente significativa entre la valoración subjetiva de los cuidadores en la ECSA (respuesta a la pregunta, “¿Está usted preocupado por el desarrollo emocional y del comportamiento del niño?") y un estado positivo de riesgo $\left(\chi^{2}=11,7 ; p<0,01\right)$.

\section{Análisis factorial exploratorio}

Mediante un análisis factorial exploratorio, se determinó la estructura interna de la prueba. Como medidas de adecuación de la muestra, se utilizaron el índice de Kaiser-Meyer-Olkin, en el cual un valor satisfactorio es mayor a 0,70, y la prueba de esfericidad de Bartlett para determinar que los ítems estuvieran correlacionados entre sí. Se utilizó una rotación ortogonal de los ítems para maximizar las ponderaciones a nivel de factor. Los resultados fueron satisfactorios, con un valor del índice de Kaiser-Meyer-Olkin de 0,78 y, en la prueba de Bartlett, un $\chi^{2}(630)$ de $1.788,38$ y $p<0,01$. Por lo tanto, las medidas de adecuación evidenciaron que el análisis factorial exploratorio de los 36 ítems enfocados en el niño era apropiado, pues todos los ítems aportaban al modelo (cuadro 5).

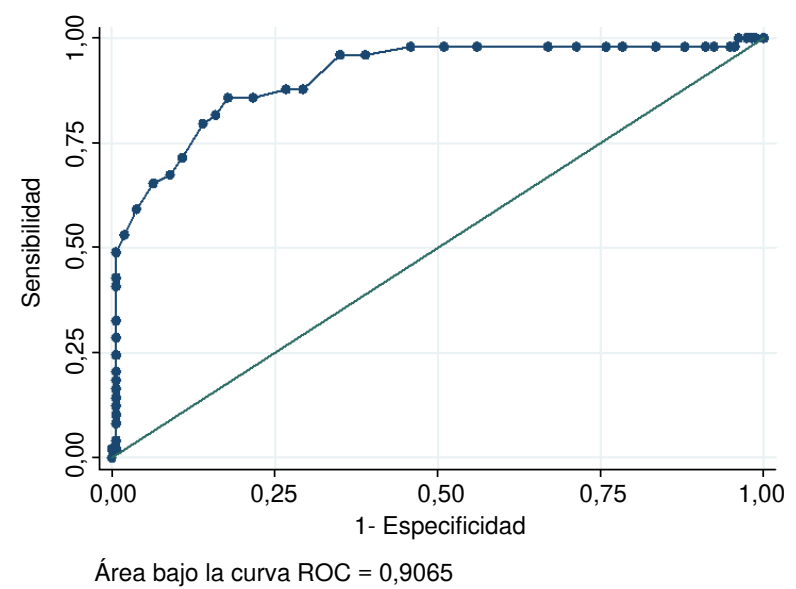

Figura 1. Características de la curva recibidor-operador 
La prueba incluyó siete factores que explicaron el $61,24 \%$ de la varianza total (como se observa en los ítems agrupados de acuerdo con el factor en el cuadro 6), teniendo en cuenta que es necesario agrupar tres ítems o más para conformar un factor en la prueba. El ítem 32 no se enmarcó en ningún factor, pero es relevante en términos de diagnóstico, ya que los enunciados se ajustaban al DSM (39). Además, el ítem hace referencia a la falta de sueño, información a todas luces relevante para la salud mental.

En el gráfico de sedimentación (figura 2), se puede observar que hay tres factores que tienen mayor peso en la prueba y que la curva tiene una menor inclinación a partir del tercer punto, lo que indica que estos factores no aportan un valor significativo a la varianza.

\section{Confiabilidad y frecuencia}

Se midió la consistencia interna del instrumento utilizando el alfa de Cronbach. La prueba tuvo una confiabilidad alta, con un alfa equivalente a 0,88, lo cual indica que todos los ítems eran relevantes para la prueba y que no se requería eliminar

Cuadro 3. Resultados de la escala ECSA

\begin{tabular}{lccc}
\hline \multirow{2}{*}{ Escala ECSA } & \multicolumn{3}{c}{$\begin{array}{c}\text { Tamización positiva según el } \\
\text { cuestionario CBCL 1,5-5 }\end{array}$} \\
\cline { 2 - 4 } & Sí & No & Total \\
\hline Positivo & 42 & 28 & 70 \\
Negativo & 7 & 129 & 136 \\
Total & 49 & 157 & 206 \\
\hline Propiedades de la ECSA & $\%$ & IC $_{95 \%}$ & \\
\hline Sensibilidad & 85,7 & $(73,3-93,0)$ & \\
Especificidad & 82,2 & $(75,4-87,4)$ & \\
Valor predictivo positivo & 60 & $(77,3-87,5)$ & \\
Valor predictivo negativo & 94,9 & $(48,3-70,7)$ & \\
Precisión del diagnóstico & 83 & $(77,3-87,5)$ & \\
Prevalencia de niños & 33,9 & $(27,5-40,9)$ & \\
en riesgo de problemas & & & \\
emocionales o del & & & \\
comportamiento & & & \\
\hline
\end{tabular}

Nota: punto de corte de 24 (>24) ninguno. Teniendo en cuenta que la escala de la prueba es de 0 a 2 (0: rara vez/no es verdad, 1: algunas veces/un poco, 2: casi siempre/muy cierto), la media de los ítems 11, 13, 14, 20, 22, 30, $32,33,35,36,37,38,39$ y 40, se situó entre 0,15 y 0,39; la de los ítems 1, 2, 6, 7, 9, 10, 12, 19, 24, 29,31 y 34 , entre 0,41 y 0,70 , y la de los ítems 3,4 , $6,8,9,15,16,17,21,23,25$ y 28 , entre 0,71 y 1 . El único ítem con una media superior a uno fue el 26 : "Siempre está haciendo algo o buscando algo para hacer", con una media de 1,52 (cuadro 7).

Se observó que los datos no estaban polarizados y no se evidenció una restricción en la variación del rango inferior (efecto suelo) ni del rango superior (efecto techo), lo que indica que no se afectó la fiabilidad de la prueba.

\section{Discusión}

Hasta donde tenemos conocimiento, no se ha desarrollado o validado una escala para la tamización de problemas emocionales y del comportamiento en la primera infancia en Colombia. Las escalas disponibles son para niños mayores de cinco años y las que incluyen una franja poblacional en edad temprana (por ejemplo, de 5 a 6 años) están diseñadas para niños en el contexto escolar. Otras escalas se centran en un dominio o trastorno específico (por ejemplo, comportamiento; trastorno por déficit de atención e hiperactividad) $(29,61)$. Es importante que los instrumentos que se utilicen en edades tempranas sean sensibles al proceso de desarrollo, por lo que es conveniente contar con un instrumento adecuado para la tamización de problemas de la primera infancia en nuestro país (11).

En el presente estudio se determinó que la traducción al español del cuestionario ECSA resultó apropiada para la tamización de problemas emocionales y del comportamiento en niños en edad temprana de estratos socioeconómicos bajo y medio. El análisis de la ROC permitió establecer el punto de corte en 24 puntos, con lo cual se maximizaron la sensibilidad y la especificidad de la prueba. Este componente en la validación inicial de la escala

Cuadro 4. Índices de validez de criterio de la escala ECSA en diferentes puntos de corte

\begin{tabular}{lcccccc}
\hline Propiedades de la escala ECSA & \multicolumn{5}{c}{ Puntos de corte } \\
\cline { 2 - 7 } & $\mathbf{2 3}$ & $\mathbf{2 4}$ & $\mathbf{2 5}$ & $\mathbf{2 6}$ & $\mathbf{2 7}$ & $\mathbf{2 8}$ \\
\hline Sensibilidad (\%) & 85,7 & 85,7 & 81,6 & 79,6 & 71,4 & 67,4 \\
Especificidad (\%) & 78,3 & 82,2 & 84,1 & 86,0 & 89,2 & 91,1 \\
Valor predictivo positivo (\%) & 55,3 & 60,0 & 61,5 & 63,9 & 67,3 & 70,2 \\
Valor predictivo negativo (\%) & 94,6 & 94,9 & 93,6 & 93,1 & 90,1 & 89,9 \\
\hline
\end{tabular}


Cuadro 5. Proporción de la varianza explicada por los factores comunes en las variables

\begin{tabular}{|c|c|c|}
\hline & Inicial & Extracción \\
\hline 1-Triste, llora & 1,000 & 0,568 \\
\hline 2- Difícil de calmar & 1,000 & 0,494 \\
\hline 3- Pierde la paciencia & 1,000 & 0,618 \\
\hline 4- Evita situaciones & 1,000 & 0,606 \\
\hline 5- Se distrae & 1,000 & 0,537 \\
\hline 6- Lastima a otros & 1,000 & 0,604 \\
\hline 7- No escucha & 1,000 & 0,527 \\
\hline 8- La comida es una lucha & 1,000 & 0,588 \\
\hline 9- Irrita/molesta & 1,000 & 0,589 \\
\hline 10- Discute & 1,000 & 0.666 \\
\hline 11- Rompe cosas & 1,000 & 0,682 \\
\hline 12- Se asusta & 1,000 & 0,558 \\
\hline 13- Fastidioso & 1,000 & 0,586 \\
\hline 14- No interactúa & 1,000 & 0,677 \\
\hline 15- Inquieto(a) & 1,000 & 0,633 \\
\hline 16-Muy apegado(a) & 1,000 & 0,604 \\
\hline 17- Mucho miedo & 1,000 & 0,416 \\
\hline 18- Nervioso(a) & 1,000 & 0,662 \\
\hline 19- Culpa a otros & 1,000 & 0,657 \\
\hline 20- Se paraliza & 1,000 & 0,532 \\
\hline 21- Rechaza alimentos & 1,000 & 0.682 \\
\hline 22- Interés sexual & 1,000 & 0,704 \\
\hline 23- Corre & 1,000 & 0,555 \\
\hline 24- No presta atención & 1,000 & 0,665 \\
\hline 25- Interrumpe & 1,000 & 0,597 \\
\hline 26- Siempre está haciendo algo & 1,000 & 0,616 \\
\hline 27- Reacciones emocionales & 1,000 & 0,648 \\
\hline 28- Desobediente & 1,000 & 0,622 \\
\hline 29- Selectivo con la comida & 1,000 & 0,723 \\
\hline 30- Comportamientos repetitivos & 1,000 & 0,660 \\
\hline 31- Camina solo(a) & 1,000 & 0,706 \\
\hline 32- No duerme & 1,000 & 0.576 \\
\hline 33- No se divierte & 1,000 & 0,448 \\
\hline 34- Amigable con extraños & 1,000 & 0,655 \\
\hline 35- No habla bien & 1,000 & 0,739 \\
\hline 36- Desarrollo lento & 1,000 & 0,647 \\
\hline
\end{tabular}

sugiere que el ECSA podría ser un instrumento adecuado para detectar un riesgo emocional o de comportamiento en niños entre el año y medio y los seis años de edad. No obstante, se recomienda hacer estudios posteriores con un tamaño de muestra mayor, para determinar si el ítem 32 puede agruparse en algún factor de la prueba.

Además, se encontró una relación entre la valoración subjetiva de los cuidadores en el ECSA (respuesta a la pregunta: "¿Está usted preocupado por el desarrollo emocional y del comportamiento de la niña o del niño?") y un estado positivo de riesgo $\left(\chi^{2}=11,7 ; p<0,01\right)$. Este resultado es valioso porque una única pregunta del cuestionario se relaciona positivamente con un estado de riesgo en los niños, lo cual permitiría considerar dicha pregunta como un primer filtro durante la tamización de sus problemas socioemocionales en cualquier nivel de atención.
Es importante resaltar que cuestionarios diligenciados por los padres, como el validado en este estudio, deben interpretarse con prudencia, primero, porque se trata de las percepciones de los cuidadores, $y$, segundo, porque la salud mental es un tema sensible que puede llevar al estigma social de los niños. Un resultado positivo en la prueba no establece un diagnóstico patológico, sino que indica un estado de riesgo que debe ser evaluado cuidadosamente por un profesional de la salud mental.

En cuanto a las limitaciones del estudio y de la herramienta, en primer lugar debe señalarse que el CBCL 1,5-5 ha sido validado en muchos ámbitos y se utiliza con frecuencia como herramienta de comparación para establecer la validez de otras escalas (52), pero no hay un grupo normativo para Colombia, por lo que en el análisis se decidió usar el llamado grupo multicultural No. 2, que corresponde al grupo promedio reportado en todas las sociedades (12) y es el recomendado por los autores del CBCL 1,5-5.

En segundo lugar, la muestra se escogió por coveniencia, por lo que es posible que los resultados no sean representativos de la población general de niños menores de 6 años. Los jardines infantiles y los centros de desarrollo infantil donde se hizo el estudio están adscritos al ICBF, por lo que no fue posible contar con participantes de instituciones privadas o de estrato socioeconómico alto. Por lo tanto, la muestra por conveniencia de las instituciones limitó el poder para reflejar la realidad del comportamiento de los problemas emocionales y de comportamiento en el estrato socioeconómico alto, aunque ello no afecta la validez externa de los resultados obtenidos, la prevalencia de riesgo de $34 \%$ en los niños del estudio no puede generalizarse.

En tercer lugar, las preguntas sobre la depresión materna no fueron comparadas con las de otro instrumento estándar, y solo se adaptaron lingüísticamente y se probaron para verificar su comprensión. Durante el proceso de adaptación, se planteó la inquietud sobre si las preguntas para las madres eran confusas, dado que no hay una introducción en la que se aclare que hacen referencia a ellas. En el diseño del ECSA original, esto se justificó planteando que dichos ítems no se presentaban separados de los concernientes a los niños para que las madres los consideraran como parte de la evaluación de sus hijos (39). Sin embargo, se decidió añadir una breve introducción a las preguntas sobre depresión materna ("Si usted es la madre, responda las siguientes preguntas."), para mejorar la comprensión de los ítems. 
Cuadro 6. Matriz de componente rotado

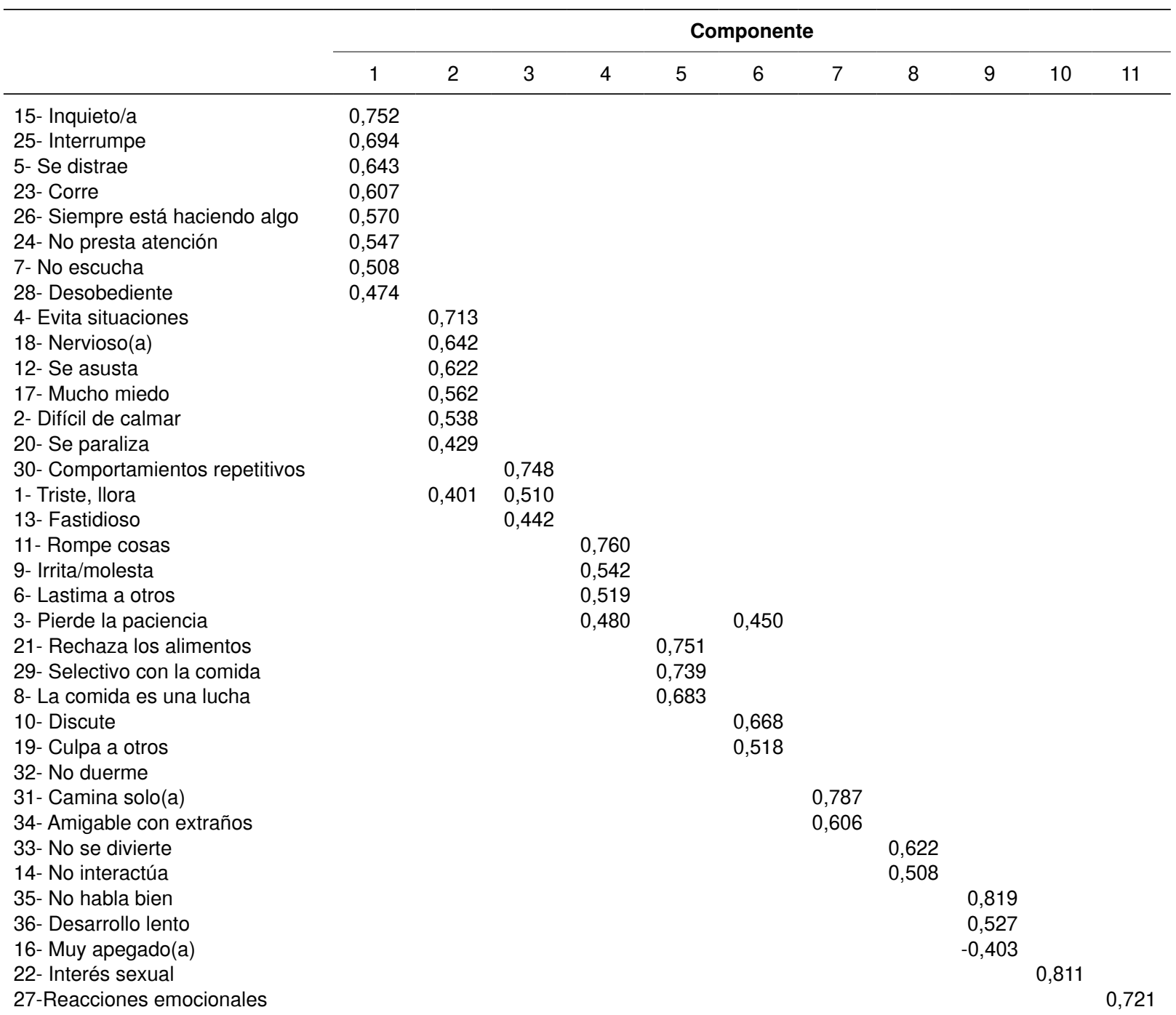

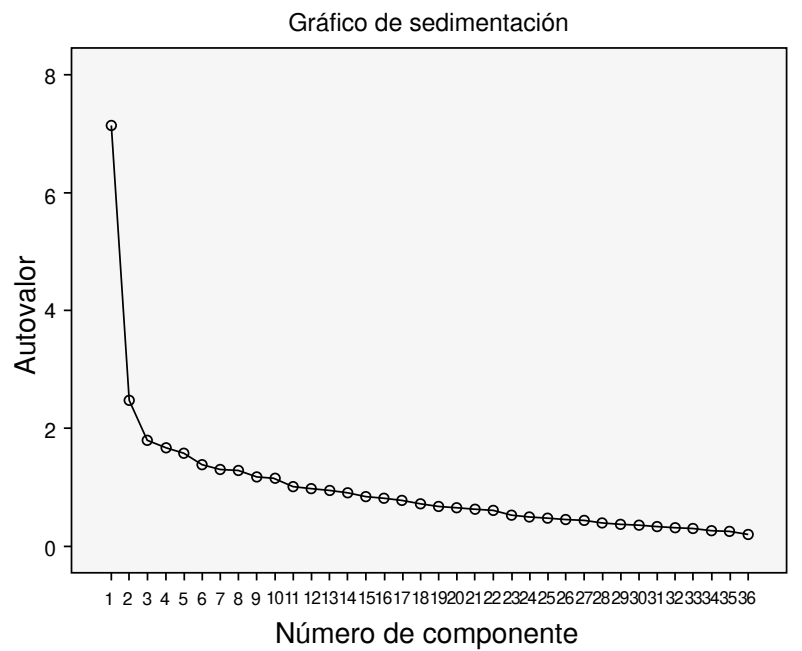

Figura 2. Gráfico de sedimentación
En este estudio se optó por la selección de un instrumento sencillo, con buenas propiedades psicométricas y que incorporara los dominios emocionales y del comportamiento. Después de revisar las diversas escalas disponibles en los artículos consultados, varios expertos en salud mental eligieron la escala más adecuada para la población y los objetivos del estudio.

En el trabajo se evidenció el potencial de la escala ECSA como herramienta de tamización, pues se registraron excelentes valores de sensibilidad, especificidad y confiabilidad. Además, es una herramienta sencilla de responder e interpretar, y está disponible gratuitamente. Según nuestros resultados, sería valioso iniciar un proceso para su uso en las instituciones educativas, acompañado de una ruta de acción para los casos positivos, lo 
Cuadro 7. Estadísticas de los elementos

\begin{tabular}{|c|c|c|c|}
\hline & Media & $\begin{array}{l}\text { Desviación } \\
\text { estándar }\end{array}$ & $\mathbf{N}$ \\
\hline 1-Triste, llora & 0,54 & 0,686 & 173 \\
\hline 2- Difícil de calmar & 0,56 & 0,603 & 173 \\
\hline 3- Pierde la paciencia & 0,87 & 0,736 & 173 \\
\hline 4- Evita situaciones & 0,77 & 0,734 & 173 \\
\hline 5- Se distrae & 0,92 & 0,694 & 173 \\
\hline 6- Lastima a otros & 0,46 & 0,703 & 173 \\
\hline 7- No escucha & 0,61 & 0,720 & 173 \\
\hline 8- La comida es una lucha & 0,73 & 0,754 & 173 \\
\hline 9- Irrita/molesta & 0,85 & 0,699 & 173 \\
\hline 10- Discute & 0,55 & 0,668 & 173 \\
\hline 11- Rompe cosas & 0,39 & 0,679 & 173 \\
\hline 12- Se asusta & 0,68 & 0,723 & 173 \\
\hline 13- Fastidioso & 0,32 & 0,588 & 173 \\
\hline 14- No interactúa & 0,21 & 0,452 & 173 \\
\hline 15- Inquieto(a) & 0,99 & 0,751 & 173 \\
\hline 16- Muy apegado(a) & 0,94 & 0,748 & 173 \\
\hline 17- Mucho miedo & 0,93 & 0,728 & 173 \\
\hline 18- Nervioso(a) & 0,42 & 0,601 & 173 \\
\hline 19- Culpa a otros & 0,68 & 0,698 & 173 \\
\hline 20- Se paraliza & 0,35 & 0,577 & 173 \\
\hline 21- Rechaza los alimentos & 0,82 & 0,713 & 173 \\
\hline 22- Interés sexual & 0,15 & 0,445 & 173 \\
\hline 23- Corre & 0,91 & 0,749 & 173 \\
\hline 24- No presta atención & 0,54 & 0,660 & 173 \\
\hline 25- Interrumpe & 0,82 & 0,688 & 173 \\
\hline 26- Siempre está haciendo algo & 1,52 & 0,625 & 173 \\
\hline 27- Reacciones emocionales & 0,42 & 0,611 & 173 \\
\hline 28- Desobediente & 0,73 & 0,700 & 173 \\
\hline 29- Selectivo con la comida & 0,51 & 0,625 & 173 \\
\hline 30- Comportamientos repetitivos & 0,16 & 0,462 & 173 \\
\hline 31- Camina solo(a) & 0,57 & 0,725 & 173 \\
\hline 32- No duerme & 0,39 & 0,624 & 173 \\
\hline 33- No se divierte & 0,36 & 0,620 & 173 \\
\hline 34- Amigable con extraños & 0,67 & 0,740 & 173 \\
\hline 35- No habla bien & 0,35 & 0,652 & 173 \\
\hline 36- Desarrollo lento & 0,27 & 0,527 & 173 \\
\hline 37- Deprimida & 0,23 & 0,499 & 173 \\
\hline 38- Falta de interés & 0,20 & 0,466 & 173 \\
\hline 39- Estresada & 0,26 & 0,546 & 173 \\
\hline 40- Frustrada & 0,29 & 0,570 & 173 \\
\hline
\end{tabular}

cual contribuiría a mejorar la salud mental de la primera infancia. Es importante resaltar que esta tamización no indica un trastorno específico en el niño, sino un estado de riesgo que, al ser atendido, permite prevenir futuras dificultades y mejorar la calidad de vida del individuo.

\section{Agradecimientos}

A Mary Margaret Gleason, por permitirnos adaptar el cuestionario y por su continua realimentación; a Francisco Lamus y José Posada Villa, por su asesoría; a Germán Casas y Roberto Chaskel, por su participación en las entrevistas y el consenso de expertos; a Maira Alejandra Soto, Liliana Betancourt, Paola Tejada y Diana Botero, y a los psicólogos Juan José Giraldo, Milton Bermúdez y Catalina Artunduaga, por su participación en el consenso de expertos; a José Cepeda, por sus aportes durante la adaptación lingüística; a Marisol Carreño, por su orientación sobre el método Delphi; a Carolina Ladino y Catalina Rico, por las traducciones del instrumento; a Mauricio Pérez y Hanner Sánchez, por su apoyo en el diseño del muestreo y el análisis estadístico, y a la Alcaldía de Tunja, la Secretaría de Educación de Sopó y la Experiencia Educativa aeioTU.

\section{Conflicto de intereses}

Los autores declaran no tener conflictos de intereses.

\section{Financiación}

El estudio fue financiado por el Departamento Administrativo de Ciencia, Tecnología e Innovación, Colciencias, convocatoria 537 de 2011, y la convocatoria 617 de 2013 del programa de Jóvenes investigadores e innovadores, marzo 2014 a febrero 2015, y se hizo en el marco del programa "Inicio parejo de vida", financiado por la Fundación Santa Fe de Bogotá, la Fundación Corona, la Organización para la Excelencia de la Salud y la Universidad de la Sabana.

\section{Referencias}

1. Kolko DG. The effectiveness of integrated care on pediatric behavioral health: Outcomes and opportunities. JAMA Pediatr. 2015;69:894-96. https://doi.org/10.1001/ jamapediatrics.2015.1428

2. Briggs-Gowan MJ, Carter AS. Social-emotional screening status in early childhood predicts elementary school outcomes. Pediatrics. 2008;121:957-62. https://doi.org/10. 1016/j. biotechadv.2011.08.021

3. Denham SA. Emotional competence: Implications for social functioning. En: Luby JL, editor. Handbook of preschool mental health: Development, disorders and treatment. 2nd edition. New York: The Guilford Press; 2009. p. 23-44.

4. McCabe PC, Altamura M. Empirically valid strategies to improve social and emotional competence of preschool children. Psychol Sch. 2011;48:513-40. https://doi.org/10. 1002/pits.20570

5. Thompson RA, Goodvin R, Meyer S. Social development: Psychosocial understanding, self-understanding, and relationships En: Luby JL, editor. Handbook of Preschool Mental Health: Development, disorders and treatment. 2nd edition. New York: The Guilford Press; 2009. p. 3-22.

6. Zero to Three Policy Center. Infant and early childhood mental health: Promoting healthy social and emotional development. Mental Health Care. 2004. Fecha de consulta: 8 de septiembre de 2015. Disponible en: http:// www.childinst.org/images/stories/resource_center_docs/ Infant_and_Early_Childhood_Mental_Health_Fact_Sheet.pdf

7. Egger HL, Angold A. Common emotional and behavioral disorders in preschool children: Presentation, nosology, and epidemiology. J Child Psychol Psychiatry. 2006;47:313-37. https://doi.org/10.1111/j.1469-7610.2006.01618.x 
8. García-Jaramillo S, Fernández-Monsalve C, SánchezTorres F. Deserción y repetición en los primeros grados de la básica primaria: factores de riesgo y alternativas de política pública. Fecha de consulta: 8 deseptiembre de 2015. Disponible en: https://egob.uniandes.edu.co/images/ books/pdf/ECT\%20Desercion\%20y\%20repeticionFINAL.pdf

9. Tomlinson M, Bornstein MH, Marlow M, Swartz L. Imbalances in the knowledge about infant mental health in rich and poor countries: Too little progress in bridging the gap. Infant Ment Health J. 2014;35:624-9. https://doi. org/10.1002/imhj.21462

10. Tomlinson M, Swartz L. Imbalances in the knowledge about infancy: The divide between rich and poor countries. Infant Ment Health J. 2003;24:547-56. https://doi.org/10.1002/ imhj.10078

11. Carter AS, Briggs-gowan MJ, Davis NO. Assessment of young children's social-emotional development and psychopathology: Recent advances and recommendations for practice. J Child Psychol Psychiatry. 2004;45:109-134. https://doi.org/ 10.1046/j.0021-9630.2003.00316.x

12. Ivanova MY, Achenbach TM, Rescorla LA, Harder VS, Ang RP, Bilenberg $\mathbf{N}$, et al. Preschool psychopathology reported by parents in 23 societies: Testing the sevensyndrome model of the child behavior checklist for ages 1.5-5. J Am Acad Child Adolesc Psychiatry. 2010;49:121524. https://doi.org/10.1016/ j.jaac.2010.08.019

13. Carter AS.The field of toddler/preschool mental health has arrived--on a global scale. J Am Acad Child Adolesc Psychiatry. 2010;49:1181-2. https://doi.org/10.1016/j.jaac. 2010.09.006

14. Luby JL. Handbook of preschool mental health: Development, disorders and treatment. 2nd edition. New York: The Guilford Press; 2009. p. 23-44.

15. National Scientific Council on the Developing Child. Mental health problems in early childhood can impair learning and behavior for life. Working Paper \# 6. Childhood A Global Journal Of Child Research. Fecha de consulta: 10 de septiembre de 2015. Disponible en: http://46y5eh11 fhgw3ve3ytpwxt9r.wpengine.netdna-cdn.com/wp-content/ uploads/2008/05/Establishing-a-Level-Foundation-for-LifeMental-Health-Begins-in-Early-Childhood.pdf

16. American Psychiatric Association. Diagnostic and statistical manual of mental disorders. Fifth edition. Washington DC: American Psychiatric Pub; 2013. p. 492.

17. Bird HR, Canino G, Rubio-Stipec M, Gould MS, Ribera J, Sesman M, et al. Estimates of the prevalence of childhood maladjustment in a community survey in Puerto Rico the use of combined measures. Arch Gen Psychiatry. 1988:45:11206. https://doi.org/10.1001/archpsyc.1988.01800360068010

18. Caraveo-Anduaga JJ, Colmenares-Bermúdez E, MartínezVélez NA. Síntomas percepción y demanda de atención en salud mental en niños y adolescentes de la Ciudad de México. Salud Pública Mex. 2002;44:492-8.

19. Lorena C, Higueros M, Alejandra M, Izazaga Á. Detección temprana de trastornos de salud mental. Diseño, validación y confiabilidad del cuestionario de salud mental en atención primaria para niños de 6 a 12 años. Revista Clínica de Medicina Familiar. 2010;3:170-6.

20. Vicente B, Saldivia S, Rioseco P, De la Barra F, Valdivia $\mathbf{M}$, Melipillan R, et al. Epidemiología de trastornos mentales infanto-juveniles en la Provincia de Cautín. Revista Médica De Chile. 2010;138:965-73. https://doi.org/10.4067/S003498872010000800004

21. Palacio JD, Ruiz-García M, Bauermeister JJ, MontielNavas C, Henao GC, Agosta G. Algoritmo de tratamiento multimodal para preescolares latinoamericanos con trastorno por déficit de atención con hiperactividad (TADH). Salud Mental. 2009;32:3-16.

22. Pineda D, Ardila A, Rosselli M, Arias BE, Henao GC, Gómez LF, et al. Prevalence of attention-deficit/ hyperactivity disorder symptoms in 4- to 17-year-old children in the general population. J Abnorm Child Psychol. 1999;27:455-62.

23. Gómez-Restrepo C, Aullí J, Tamayo-Martínez N, Gil F, Garzón D, Casas G. Prevalencia y factores asociados a trastornos mentales en la población de niños colombianos. Encuesta Nacional de Salud Mental (ENSM), 2015. Revista Colombiana de Psiquiatría. 2016;45:39-49. https://doi.org/10. 1016/j.rcp.2016.06.010

24. Carlson VJ, Hardwood RL. Attachment, culture, and the caregiving system: The cultural patterning of everyday experiences among Anglo and Puerto Rican mother-infant pairs. Infant Ment Health J. 2003;24:53-73. https://doi. org/10.1002/imhj.10043

25. Lecannelier F, Silva JR, Hoffmann M, Melo R, Morales R. Effects of an intervention to promote socio-emotional development in terms of attachment security: A study in early institutionalization in Chile. Infant Ment Health $\mathrm{J}$. 2014;35:151-9. https://doi.org10.1002/imhj.21436

26. McCall R, Groark LF, Harkins D, Serrano G, Gordon K. A socio-emotional intervention in a Latin American orphanage. Infant Ment Health J. 2010;31:521-42. https:// doi.org/10.1002/imhj.20270

27. Pineda D, Lopera F, Henao G, Palacio J, Castellanos F. Confirmación de la alta prevalencia del trastorno por déficit de atención en una comunidad colombiana. Rev Neurol. 2001;32:217-22.

28. Sandoval JJ. Ambiente escolar, familiar y comunitario en relación con los comportamientos agresivos y prosociales en niños de 3 a 12 años, Medellín, Colombia, 2001. Rev Fac Nac Salud Pública. 2006;24:30-9.

29. Giel R, De Arango MV, Climent CE, Harding TW, Ibrahim HHA, Murthy RS, et al. Childhood mental disorders in primary health care: Results of observations in four developing countries. Pediatrics. 1981;68:677-83.

30. Hernández-Barrera AB, Restrepo-Espinosa MH. Salud mental en niños en condición de desplazamiento en Colombia y su incidencia en políticas públicas. Fecha de consulta: 10 de octubre de 2016. Disponible en: http:// repository.urosario.edu.co/handle/10336/2693

31. Rutter M, Bishop D, Pine D, Scott S, Stevenson J, Taylor E, et al. Rutter's Child and Adolescent Psychiatry. $5^{\text {th }}$ edition. Oxford: Blackwell; 2008.

32. Briggs RD, Stettler EM, Silver EJ, Schrag RD, Nayak M, Chinitz S, et al. Social-emotional screening for infants and toddlers in primary care. Pediatrics. 2012;129:e377-e84. https://doi.org/10.1542/peds.2010-2211

33. Goodman R, Ford T, Simmons H, Gatward R, Meltzer H. Using the Strengths and Difficulties Questionnaire (SDQ) 
to screen for child psychiatric disorders in a community sample. Br J Psychiatry. 2000;177:534-9. https://doi.org/10. 1192/bjp.177.6.534

34. Sheldrick RC, Merchant S, Perrin EC. Identification of developmental-behavioral problems in primary care: A systematic review. Pediatrics. 2011;128:356-63. https://doi. org/10.1542/peds.2010-3261

35. Skovgaard A. Mental health problems and psychopathology in infancy and early childhood. An epidemiological study. Dan Med Bull. 2010;57:B4193.

36. Montero I, León OG. A guide for naming research studies in Psychology. Int J Clin Health Psychol. 2007;7:874-62.

37. Jellinek MS, Murphy JM, Robinson J, Feins A, Lamb S, Fenton T. Pediatric Symptom Checklist: Screening school-age children for psychosocial dysfunction. J Pediatr. 1988;112:201-9.

38. Squires J, Bricker D, Twombly E. The ASQ: SE User's Guide for the Ages \& Stages Questionnaires, socialemotional: A parent completed, child-monitoring system for social-emotional behaviors. Baltimore: Paul H. Brookes Publishing Co; 2003. p. 93.

39. Gleason MM, Zeanah CH. Recognizing young childern in need of mental health assessment: Development and preliminary validity of the early childhood screening assessment. Infant Ment Health J. 2010;31:335-7. https:// doi.org/10.1002/imhj.20259

40. Carreño M. El método Delphi: cuando dos cabezas piensan más que una en el desarrollo de guías de práctica clínica. Revista Colombiana de Psiquiatría. 2009;38:185-93.

41. Cuhls K. Delphi method. Fecha de consulta: 3 de agosto de 2014. Disponible en: www.unido.org/fileadmin/import/ 16959_Delphi Method.pdf

42. Hasson F, Keeney S, McKenna H. Research guidelines for the Delphi survey technique. J Adv Nurs. 2000;32:1008-15. https://doi.org/10.1046/j.1365-2648.2000.t01-1-01567.x

43. Hsu CC, Sandford BA. The Delphi technique: Making sense of consensus. Practical Assessment, Research \& Evaluation. 2007;12:1-8.

44. Beaton DE, Bombardier C, Guillemin F, Ferraz MB. Guidelines for the process of cross-cultural adaptation of self-report measures. Spine. 2000;25:3186-91.

45. Gjersing L, Caplehorn JRM, Clausen T. Cross-cultural adaptation of research instruments: Language, setting, time and statistical considerations. BMC Med Res Methodol. 2010;10:13. https://doi.org/10.1186/1471-2288-10-13

46. Oquendo-Jiménez I, Mena R, Antoun MD, Wojna V. Linguistic adaptation of the clinical dementia rating scale for a Spanish-speaking population. P R Health Sci J. 2010;29:102-8.

47. Sidani S, Guruge S, Miranda J, Ford-Gilboe M, Varcoe C. Cultural adaptation and translation of measures: An integrated method. Res Nurs Health. 2010;33:133-43. https://doi.org101002/nur.20364
48. Sousa VD, Rojjanasrirat W. Translation, adaptation and validation of instruments or scales for use in cross-cultural health care research: A clear and user-friendly guideline. J Eval Clin Pract. 2011;17:268-74. https://doi.org/10.1111/ j.1365-2753.2010.01434.x

49. Weiner J. Measurement: Reliability and validity measures, Johns Hopkins Bloomberg School of Public Health. Fecha de consulta: 17 de julio de 2014. Disponible en: http://ocw. jhsph.edu/courses/hsre/PDFs/HSRE_lect7_weiner.pdf

50. Argibay J. Técnicas psicométricas. Cuestiones de validez y confiabilidad. Subjetividad y Procesos Cognitivos. 2006; 8:15-33.

51. Rattray J, Jones MC. Essential elements of questionnaire design and development. J Clin Nurs. 2007;16:234-43. https://doi.org/10.1111/j.1365-2702.2006.01573.x

52. Muzzolon SR, Nunes M, Dos Santos LH. Evaluación del uso de la lista de síntomas pediátricos como instrumento de rastreo para identificar problemas emocionales y psicosociales. Rev Paul Pediatr. 2013;31:359-65. https://doi. org/10.1590/S0103-05822013000300013

53. Achenbach T. ASEBA- Achenbach System of Empirically Based Assessment. Fecha de consulta: 21 de mayo de 2014. Disponible en: http://www.aseba.org/products/ asebabook.html?dept=64

54. Zárate O, López C, Flores G. Adaptación del cuestionario sobre el comportamiento en niños de $1 \frac{1 / 2}{2}$ a 5 años de Achenbach para niños de 4 a 5 años. Enseñanza e Investigación en Psicología. 2009;14:179-91.

55. Achenbach T, Rescorla L. Manual for the ASEBA preschool form and profiles. Burlington: University of Vermont; 2000. p. 14.

56. Shapiro SS, Wilk MB. An analysis of variance test for normality (Complete Samples). Biometrika. 1965;52:591611. https://doi.org/10.2307/2333709

57. Mee RW. Confidence intervals for probabilities and tolerance regions based on a generalization of the Mann-Whitney statistic. J Am Stat Assoc. 1990;85:793-800.

58. Arribas MC. Diseño y validación de cuestionarios. Matronas Profesión. 2004;5:23-9.

59. O'Hara R, Kraemer H, Yesavage J, Thompson J, Noda A, Taylor J, et al. Receiver operating characteristic curve (ROC): Analysis for prediction studies. Fecha de consulta: 9 de junio de 2014. Disponible en: http://www.stanford. edu/ yesavage/ROC\%20Slides\%20OHara.ppt\#1

60. Masson LF, McNeil G, Tomany JO, Simpson JA. Statistical approaches for assessing the relative validity of a foodfrequency questionnaire: Use of correlation coefficients and the kappa statistic. Public Health Nutr. 2003;6:313-21. https://doi.org/10.1079/PHN2002429

61. Pineda D, Aguirre DC, García M, Lopera FJ, Palacio LG, Kamphaus RW. Validation of two rating scales for attention-deficit hyperactivity disorder diagnosis in Colombian children. Pediatr Neurol. 2005;33:15-25. https://doi. org/10.1016/j.pediatrneurol.2005.02.001 